\title{
Measurement of local relative displacements in large structures
}

\author{
Tesauro, Angelo; Eder, Martin Alexander; Nielsen, Magda
}

Published in:

Journal of Strain Analysis for Engineering Design

Link to article, DOI:

$10.1177 / 0309324713519622$

Publication date:

2014

Link back to DTU Orbit

Citation (APA):

Tesauro, A., Eder, M. A., \& Nielsen, M. (2014). Measurement of local relative displacements in large structures. Journal of Strain Analysis for Engineering Design, 49(5), 301-314. https://doi.org/10.1177/0309324713519622

\section{General rights}

Copyright and moral rights for the publications made accessible in the public portal are retained by the authors and/or other copyright owners and it is a condition of accessing publications that users recognise and abide by the legal requirements associated with these rights.

- Users may download and print one copy of any publication from the public portal for the purpose of private study or research.

- You may not further distribute the material or use it for any profit-making activity or commercial gain

- You may freely distribute the URL identifying the publication in the public portal

If you believe that this document breaches copyright please contact us providing details, and we will remove access to the work immediately and investigate your claim 


\title{
Measurement of local relative displacements in large structures
}

\author{
A. Tesauro, M. A. Eder, M. Nielsen \\ Technical University of Denmark, Department of Wind Energy, Frederiksborgvej 399, 4000 Roskilde, Denmark
}

\begin{abstract}
This paper presents a novel measurement technique to measure local relative displacements between parts of large-scale structures. The measured deformations can be of significant importance for fracture analyses in many different types of structures in general, and for adhesive connections in particular. The measurement of small local relative displacements in structures subjected to large global deformations is complex and hardly feasible with conventional measurement methods. Therefore, a Small Displacement Measurement System (SDMS) has been devised. The SDMS is based on stereo photogrammetry and capable of measuring 3D local displacements with a high degree of accuracy. In this article, the technique is used to measure local deformations in the vicinity of the adhesive trailing edge joint of a wind turbine rotor blade. The SDMS results correspond well with another independent measurement method.
\end{abstract}

\section{Keywords}

Small displacement measurement system (SDMS), Stereo photogrammetry, local relative displacement (LRD), trailing edge, wind turbine blade

\section{Correspondence}

Martin Eder, DTU, Department of Wind Energy, Frederiksborgvej 399, 4000 Roskilde, Denmark

E-mail: maed@dtu.dk, Mobile: +4521798834

\section{Introduction}

Fracture analysis of large structures - especially those made from composite materials - has achieved considerable significance in many fields of applied engineering e.g. naval, aeronautical and civil engineering. Wind turbine rotor blades are a typical representative of these class of structures and are commonly manufactured from glass fibre reinforced composites. Most manufacturers produce the blade in separate parts which are eventually assembled and bonded together by several adhesive joints (see Fig. 1b).

One important joint occurs along the trailing edge where the air flow around the airfoil re-joins and leaves the 


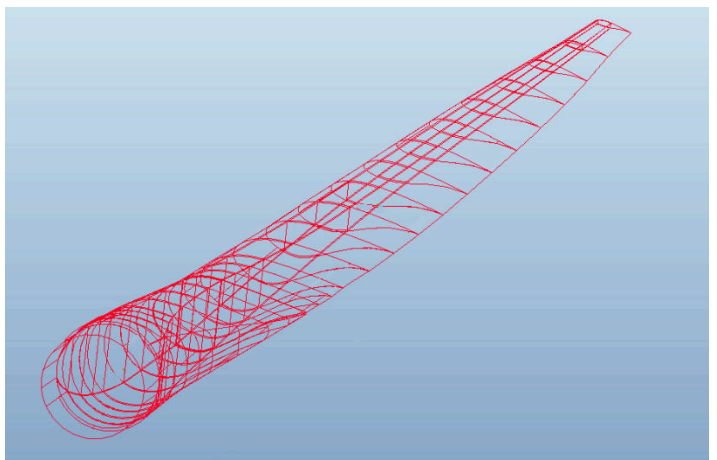

(a)

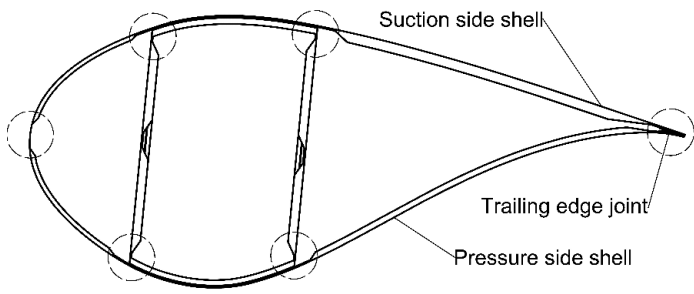

(b)

Figure 1: 3D drawing of a typical wind turbine blade, (a). The structure is composed by an external aerodynamic shell glued onto an internal load carrying spar. The cross section (airfoil) is shown in (b) with adhesive joints indicated by circles.

wind turbine blade. Proprietary inspection reports of wind turbine blades indicate that adhesive trailing edge joints are affected by various forms of damage that diminish the aerodynamic performance, decrease the life span of the blade and can even represent a threat for the structural integrity of the whole turbine. Figure 2a shows a complete separation of the two shells along the bond line of the trailing edge. Figure $2 \mathrm{~b}$ shows local buckling induced trailing edge failure in a blade test conducted at the DTU testing facility.

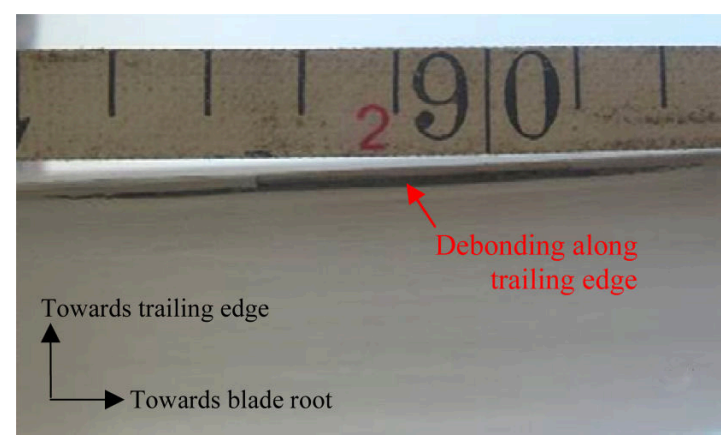

(a)

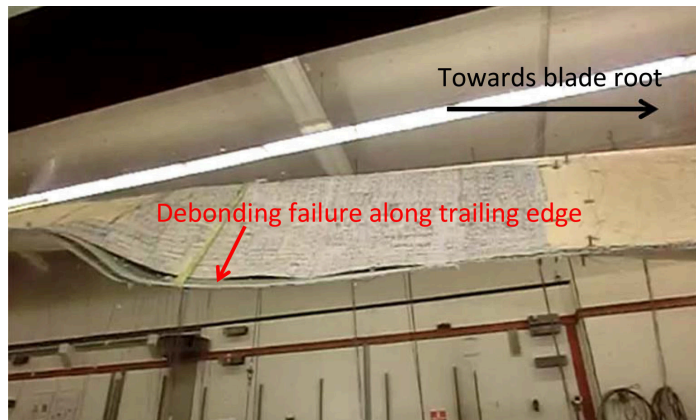

(b)

Figure 2: Typical debonding between pressure side and suction side shell at the trailing edge taken from [3]. The debonding along the trainling edge is depicted by the dark area on the shell (a). Local buckling induced trailing edge failure (b)

A strong research demand exists on the identification of the driving fracture mechanisms in the adhesive layer of the trailing edge. A well-established approach for blade designers is the adoption of the finite element method (FEM) to conduct fracture analysis of adhesive joints. In the realm of fracture analysis the availability of experimental data of the local relative displacement (LRD) in the vicinity of adhesive joints is of significant importance for validation of the numerical models and for interpretation purposes. To the authors best knowledge literature 
does to date not provide any experimental LRD data taken at trailing edges of wind turbine blades. The lack of such experimental data and the deficiency of a suitable measurement technique able to measure LRDs at trailing edges stirred the development of the Small Displacement Measurement System (SDMS).

To clarify the reasons for the SDMS development, a brief description of the fracture mechanical considerations is given. Figure 3a depicts a section of a typical trailing edge joint which shows that the suction and pressure side shell meet at an acute angle.

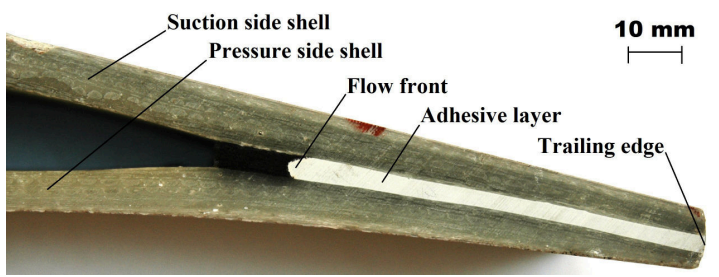

(a)

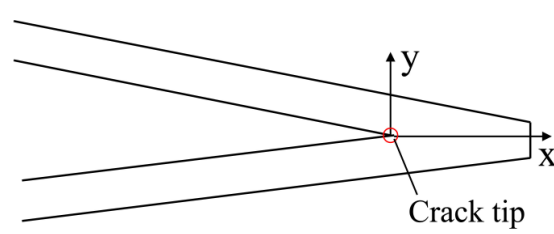

(b)

Figure 3: Section of an adhesive joint at trailing edge (a) and its simplification (b)

Figure $3 \mathrm{~b}$ shows that the situation can be simplified as a re-entrant corner at the flow front of the adhesive which leads to stress concentrations and consequently to the initiation of cracks. Moreover, initial cracks in the form of flaws such as chemically debonded areas or entrapped air bubbles are likely to be inherently present in the adhesive bond line. It is therefore reasonable to adopt the re-entrant corner as crack tip and to define a local crack coordinate system as shown in Fig. $3 \mathrm{~b}$ and 4 . There are three different modes that can cause a crack to propagate in the $x$ direction (see Fig. 4).
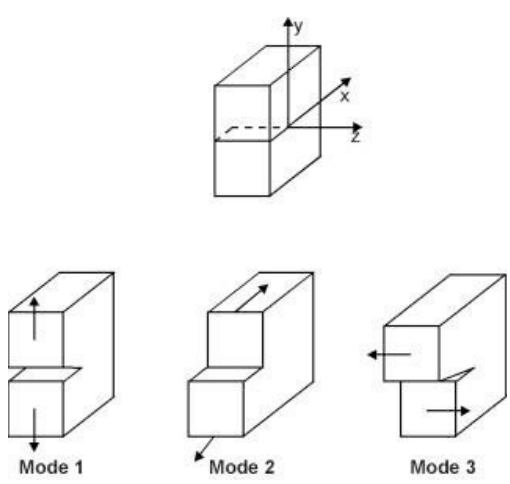

Figure 4: Fracture modes Mode-I (opening), Mode-II (in-plane shear) and Mode-III (out of plane shear) taken from $[2]$

Mode-I refers to an LRD associated with the $y$ direction, Mode-II is associated with an LRD in the $x$ direction and Mode-III is associated with an LRD in the $z$ (out of plane) direction. Inferring from Fig. 4, the measured 
LRD components between the two trailing edge shells close to the flow front can be used to give an indication of the mode mixity and the governing fracture mode involved. It needs to be stressed that the modal crack deformations of the crack mouth depicted in Fig. 4 are LRDs. In order to be consistent with fracture mechanics, a method is required which is capable of measuring the LRDs of the two shells close to the flow front in a local coordinate system that follows the deformation of the structure. The measurement of LRDs in large structures is in general rather complex and can be done either external from the structure or internal on the structure.

In the external approach the measurement equipment is located remote from the structure. Initially the global deformations are obtained e.g. by digital image correlation and subsequently the LRDs are obtained by subtraction of the rigid body motions (RBMs). The external approach raises two issues; 1) The LRD can be two to three orders of magnitude smaller than the RBM which means that the demands on the accuracy of the measurement instrument are high. This constrains the position of the measurement equipment to be in close vicinity of the measurement area, which requires repositioning of the equipment when large global deformations are concerned. 2) The RBM is a theoretical immeasurable quantity which depends on its definition following the Euler-Bernoulli approximation or Timoshenko beam theory [10]. That is to say that the extraction of RBMs from global deformations e.g. requires a plane curve fit which introduces a considerable degree of uncertainty. Hence, subtraction of biased RBMs from global deformation diminishes the precision of the LRMs.

LRDs of prismatic composite beams with open and closed cross sections subjected to torsion and bending have been measured externally by Fedorov [6] by means of a digital image correlation measurement system called Aramis [7]. Assumptions concerning RBMs in simple beam theory allowed Fedorov to extract the shapes and amplitudes of the LRDs. However, the extraction of the RBMs in structures with complex geometry and material behaviour such as wind turbine blades introduces a considerable degree of uncertainty.

In the internal approach on the other hand, the measurement device is directly mounted on the structure. In this case the measurement device follows the RBMs in such a way that the LRDs can directly be measured. Clearly, this approach is blind to any information on the components of the RBMs. Since crack growth is not associated with RBMs the latter is not considered relevant for fracture in adhesive joints. Sørensen and Jacobsen [9] used this approach to measure 2D Mode-I and Mode-II LRDs of cracks in double cantilever beam specimens using linear transducers. The internal approach is considered as more reliable and controlable when compared to the external approach and was therefore adopted by the authors.

This paper presents the principle and the functionality of the SDMS which is capable of measuring LRDs in three dimensions. Thereafter SDMS measurements taken at the trailing edge of a full scale blade tests are presented. These measurements are compared with complimentary benchmark measurements. The paper concludes with some general observations and future applications of the SDMS. 


\section{Methodology}

\subsection{LRD measurement principle}

The trailing edge measurement technique is based on three key points named as $P_{1}, P_{2}$ and $R$ in their undeformed configuration (see Fig. 5). As will be explained later, point $P_{2}$ refers to the pin mounting point located on the outer surface of the pressure side shell. Point $P_{1}$ refers to the SDMS mounting point which is defined as the intersection of the outward normal of point $P_{2}$ with the outer surface of the suction side shell. Two tangential planes $\Pi_{1}$ and $\Pi_{2}$ passing through points $P_{1}$ and $P_{2}$ can be used to define an intermediate plane $\Pi_{0}$ that bisects $\Pi_{1}$ and $\Pi_{2}$ and which passes through their line of intersection. The latter defines the alignment of the local measurement coordinate system denoted by tilde. The so called true point $R$ is defined as the projection of $P_{2}$ perpendicular to $\Pi_{0}$ onto $\Pi_{1}$ in the undeformed configuration. The purpose of $\Pi_{0}$ and $R$ is explained in more detail in the Appendix. The angle $\theta$ between planes $\Pi_{1}$ and $\Pi_{2}$ is measured in the bisector plane $\Pi_{b i}$ defined by points $P_{1}, P_{2}$ and $R$ whose outward normal direction corresponds to the line of intersection.

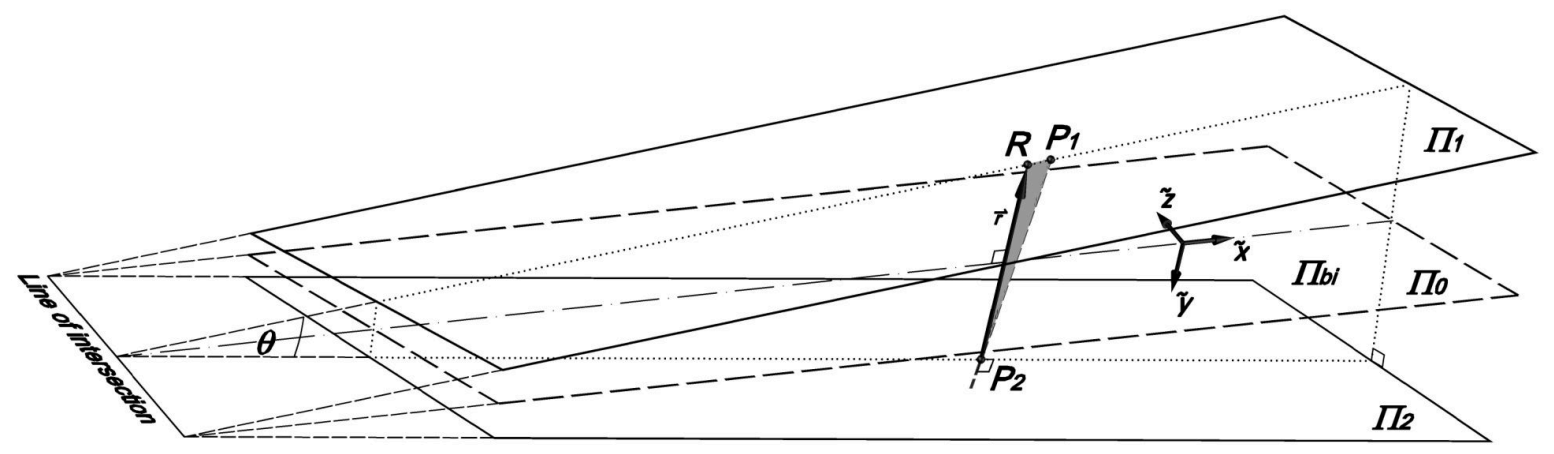

Figure 5: Definition of tangential planes $\Pi_{1}$ and $\Pi_{2}$ with intermediate plane $\Pi_{0}$ in the undeformed configuration. The grey hatched triangle defines the bisector plane whose outward normal direction is given by the line of intersection.

Figure 6 shows a $2 \mathrm{D}$ section in $\Pi_{b i}$ through the trailing edge in its undeformed and deformed configuration. Upon deformation the key points move into positions $P_{1}^{\prime}, P_{2}^{\prime}$ and $R^{\prime}$ with the right handed $\Pi_{0}$-coordinate sytem following. The axis orientation was chosen to be consistent with the image (pixel) coordinate system mentioned in the subsequent section. The LRD is defined as the mutual displacement between points $P_{2}$ and $R$ with the distance of $R$ being fixed in such a way that $\overline{P_{1} R}=\overline{P_{1}^{\prime} R^{\prime}}$. Figure $6 \mathrm{~b}$ shows that the LRD can be obtained according to equation 1.

$$
\tilde{\vec{\omega}}=\vec{r}^{\prime}-\vec{r}=\left(\tilde{\overrightarrow{\vec{R}}^{\prime}}-\tilde{\overrightarrow{P_{2}^{\prime}}}\right)-\left(\tilde{\vec{R}}-\tilde{\overrightarrow{P_{2}}}\right)
$$




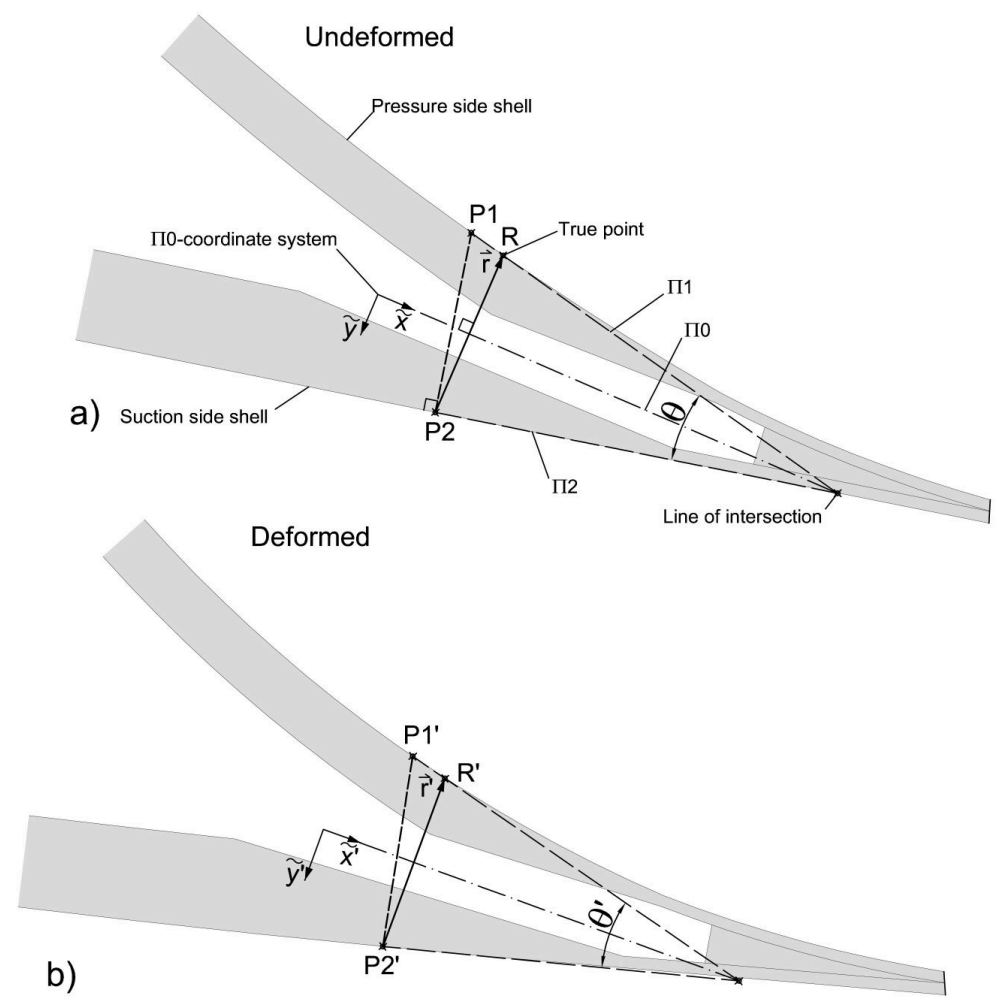

Figure 6: $2 \mathrm{D}$ section in $\Pi_{b i}$ through trailing edge joint in undeformed and deformed configuration. Tangential planes $\Pi_{1}, \Pi_{2}$ and $\Pi_{0}$ appear as line projections. The $\tilde{z}$-axis of the accompanying $\Pi_{0}$-coordinate system points in the out of plane direction.

Figure 7 shows the key points with their associated tangential planes. The origin of the measurement system

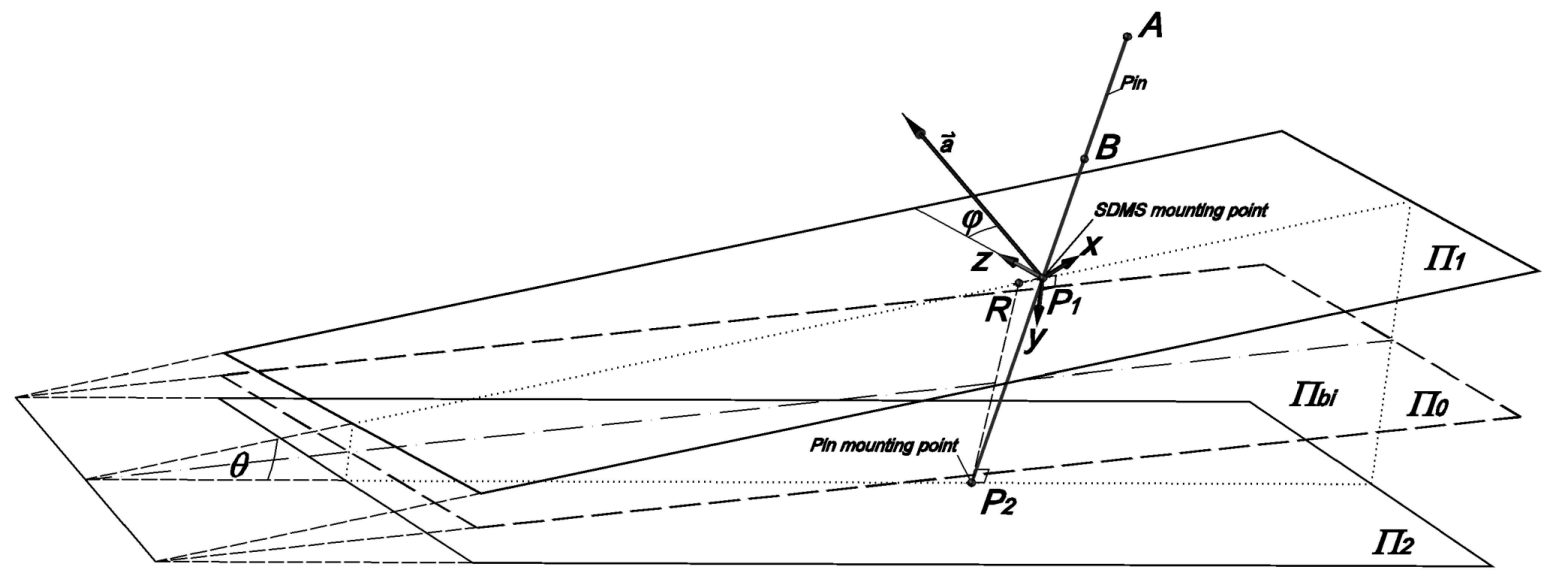

Figure 7: SDMS coordinate system and rigid pin in the undeformed configuration. The vector $\vec{a}$ defines the direction of the line of intersection. 
(i.e. SDMS coordinate system) is attached to the top shell in point $P_{1}$ in such a way that its y-axis defines the outward normal of $\Pi_{1}$. A pin is rigidly connected to the lower surface in point $P_{2}$. The purpose of the pin is to transfer information from point $P_{2}$ to the opposite side where the SDMS is located. Furthermore, the pin defines the outward normal direction of the tangential plane $\Pi_{2}$ of its mounting point.

Figure 8 shows a 2D section analogous to Fig. 6 . A borehole in the top shell with a diameter greater than that of the pin allows the latter to move independently with respect to the local coordinate system. Points $A$ and $B$ represent target points that are located on the pin axis whose coordinates can be measured. It is assumed that orthogonality between the axes and their tangents is preserved during deformation (i.e. rigid connection).

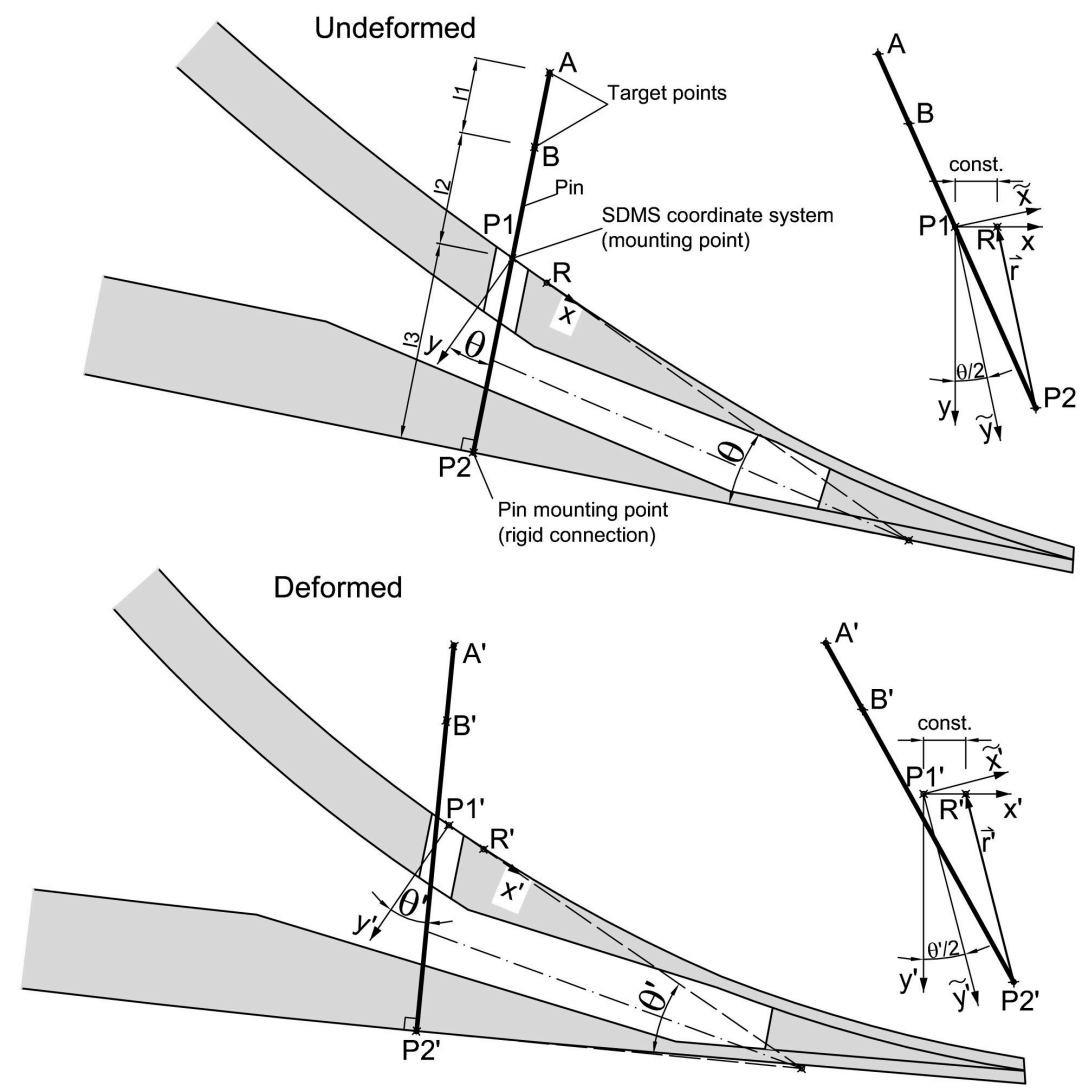

Figure 8: LRD measurement principle in 2D

In the SDMS coordinate system the outward normal of $\Pi_{1}$ is aligned with the y-axis such that $\overrightarrow{n_{1}}=[0,-1,0]^{T}$. The outward normal of $\Pi_{2}$ can be found by utilising the target points $A$ and $B$ such that $\overrightarrow{n_{2}}$ of $\Pi_{2}$ yields $\overrightarrow{n_{2}}=(\vec{A}-\vec{B}) / l_{1}$ where $l_{1}$ denotes the distance between $\overline{A B}$. Consequently, the mutual angle between $\Pi_{1}$ and $\Pi_{2}$ is given by $\cos \theta=\overrightarrow{n_{1}} \cdot \overrightarrow{n_{2}}$. The direction vector $\vec{a}=\overrightarrow{n_{1}} \times \overrightarrow{n_{2}} /\left|\overrightarrow{n_{1}} \times \overrightarrow{n_{2}}\right|$ of the line of intersection between tangential planes $\Pi_{2}$ and $\Pi_{1}$ is given by the cross product of their outward normals. The angle between $\vec{a}$ and 
the z-axis of the SDMS coordinate system in $\Pi_{1}$ is given by $\cos \varphi=\vec{a} \cdot[0,0,1]^{T}$. The true point does not change position in the SDMS coordinate system where its coordiantes can be obtained by equation 2. The coordinates of points $P_{2}$ and $P_{2}^{\prime}$ in the SDMS coordinate system can be obtained by equation 3 and 4 .

$$
\begin{gathered}
\vec{R}=\overrightarrow{R^{\prime}}=\vec{A}-\frac{l_{1}+l_{2}}{l_{1}}(\vec{A}-\vec{B})-l_{3}\left[\sin \frac{\theta}{2} \cos \varphi / \cos \frac{\theta}{2}, 0,-\sin \frac{\theta}{2} \sin \varphi / \cos \frac{\theta}{2}\right]^{T} \\
\overrightarrow{P_{2}}=\vec{A}-\frac{l_{1}+l_{2}+l_{3}}{l_{1}}(\vec{A}-\vec{B}) \\
\overrightarrow{P_{2}^{\prime}}=\vec{A}^{\prime}-\frac{l_{1}+l_{2}+l_{3}}{l_{1}}\left(\vec{A}^{\prime}-\vec{B}^{\prime}\right)
\end{gathered}
$$

where $l_{2}$ denotes the distance between $\overline{B P_{1}}$ and $l_{3}$ denotes the distance between $\overline{P_{1} P_{2}}$

The desired LRD vector in $\Pi_{0}$-coordinates can be obtained by equations 5 and 6 :

$$
\begin{gathered}
\tilde{\vec{\omega}}=[T]^{\prime}\left(\vec{R}^{\prime}-{\overrightarrow{P_{2}}}^{\prime}\right)-[T]\left(\vec{R}-\overrightarrow{P_{2}}\right) \\
{[T]=\left[T_{2}\right]\left[T_{1}\right]=\left[\begin{array}{ccc}
\cos \frac{\theta}{2} & \sin \frac{\theta}{2} & 0 \\
-\sin \frac{\theta}{2} & \cos \frac{\theta}{2} & 0 \\
0 & 0 & 1
\end{array}\right]\left[\begin{array}{ccc}
\cos \varphi & 0 & -\sin \varphi \\
0 & 1 & 0 \\
\sin \varphi & 0 & \cos \varphi
\end{array}\right]}
\end{gathered}
$$

where prime denotes entities of the deformed configuration.

Equation 6 transforms the SDMS coordinate system into the $\Pi_{0}$-coordinate system (denoted by tilde) by two subsequent rotations. First, the transformation matrix $\left[T_{1}\right]$ rotates the SDMS coordinate system by $\varphi$ around the $\mathrm{y}$-axis such that the z-axis points in the direction of $\vec{a}$. Second, the transformation matrix $\left[T_{2}\right]$ rotates the coordinate system in $\Pi_{b i}$ by $\theta / 2$ around the new z-axis.

\subsection{Software and hardware components of the SDMS}

Software based on stereo photogrammetry developed by Sheer [8] was used to obtain the position vectors $\vec{A}$ and $\vec{B}$ in the local SDMS coordinate system. Following the pin hole camera model, the projection of a point in 3D real world coordinates $\mathbf{X}=\left[\begin{array}{lll}X & Y & Z\end{array}\right]^{T}$ into $2 \mathrm{D}$ image (pixel) coordinates $u$ and $v$ can be written as follows:

$$
\begin{gathered}
u=-f \frac{\left(\boldsymbol{X}-\boldsymbol{X}_{0}\right)^{T} m_{x}}{\left(\boldsymbol{X}-\boldsymbol{X}_{0}\right)^{T} m_{s}} \\
v=-\sigma f \frac{\left(\boldsymbol{X}-\boldsymbol{X}_{0}\right)^{T} m_{y}}{\left(\boldsymbol{X}-\boldsymbol{X}_{0}\right)^{T} m_{s}}
\end{gathered}
$$

where $m_{x}, m_{y}$ and $m_{z}$ are parameters associated with the three extrinsic camera rotations in space; $\mathbf{X}_{0}=$ $\left[\begin{array}{lll}X_{0} & Y_{0} & Z_{0}\end{array}\right]^{T}$ represents the coordinates of the camera centre; $f$ and $\sigma$ denote intrinsic parameters representing 
the focal length and the aspect ratio respectively.

The eight unknowns mentioned above can be obtained by solving equations 7 und 8 using at least four calibration points with known real world coordinates and their associated image coordinates. This step is commonly also referred to as camera calibration. The maping of image coordinates into 3D real world coordinates from one image is not uniquely defined. Therefore, in order to obtain arbitrary 3D real world coordinates, a pair of digital photographs of an object taken from two different angles is required. After camera calibration using two images, equations 7 and 8 can be used to obtain the three unknown real world coordinates $\mathbf{X}=\left[\begin{array}{lll}X & Y\end{array}\right]^{T}$ by solving a set of three equations using the image coordinates on both images which represent the desired point in the real world. The accuracy of the camera calibration depends on the quality of the adopted calibration points. Although camera calibration can be performed with four calibration points, a greater number of calibration points reduces the risk of bias caused by pixel tracking (see Section 2.3).

Figure 9a depicts the components of the SDMS which consists of a square aluminium frame with an outer dimension of $170 \mathrm{~mm}$ made from tubular square sections (SHS 20x20x2).

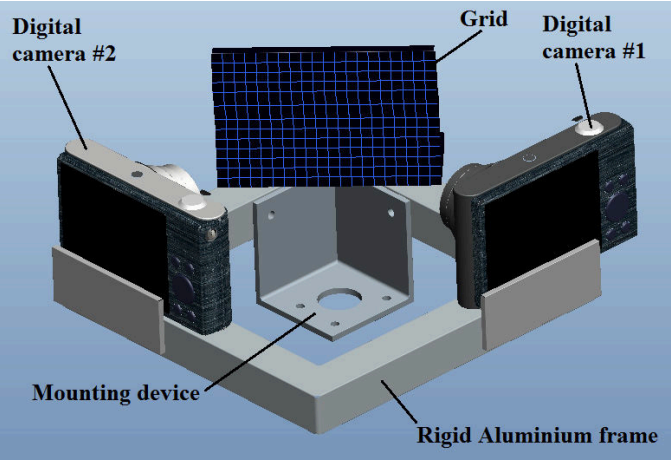

(a)

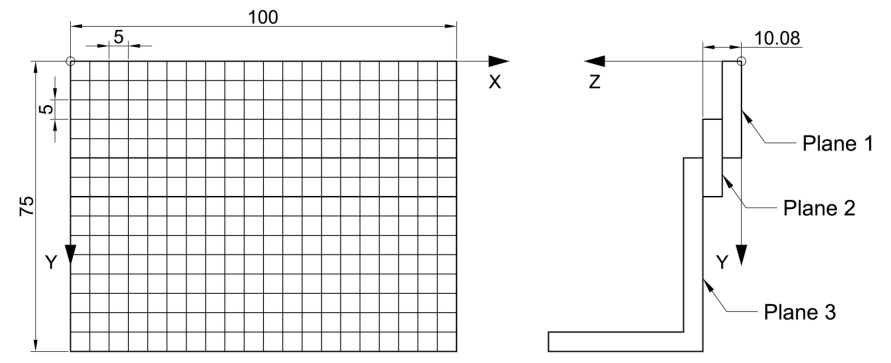

(b)

Figure 9: Hardware parts of the SDMS; Rigid frame with grid and cameras (a) Front and side elevation of the grid (b)

Two SAMSUNG ST200 digital cameras with a CMOS sensor and a resolution of 12 megapixels are perpendicularly mounted on the frame. The SAMSUNG camera automatically rectifies images taken in the macro mode and the maximum lens distortion observed was about two pixels. A square grid measuring 100x50mm with a distance of $5 \mathrm{~mm}$ between the grid centre-lines is engraved on three staggered planes of a machined and black anodised Aluminium L-section. The pitch has an accuracy of \pm 1.0 microns where the thickness of the engraved grid line measures about 100 microns. The L-section with the grid is bolted to the frame at an angle of $45^{\circ}$ in such a way that the centre of the grid is near the intersection of the optical camera axes. Figure $9 \mathrm{~b}$ shows the grid of the SDMS in its local coordinate system, where the true coordinates of the grid line intersections can be 
used for camera calibration. The fully assembled SDMS weighs $785 \mathrm{~g}$. A Tungsten pin with a diameter of $4 \mathrm{~mm}$ is firmly connected to a recessed $5 \mathrm{~mm}$ thick aluminium strip (referred to as target) in such a way that the pin axis is coplanar with the target surface of the plate. A double cross hair with a distance of $l_{1}=30 \mathrm{~mm}$ is engraved on the target surface in alignment with the pin axis. The target is adhesively connected to the bottom shell in such a way that a rigid connection can reasonably be assumed (see Fig. 10). SDMS and pin are assembled in such a way that the target is closely located in front of the grid.

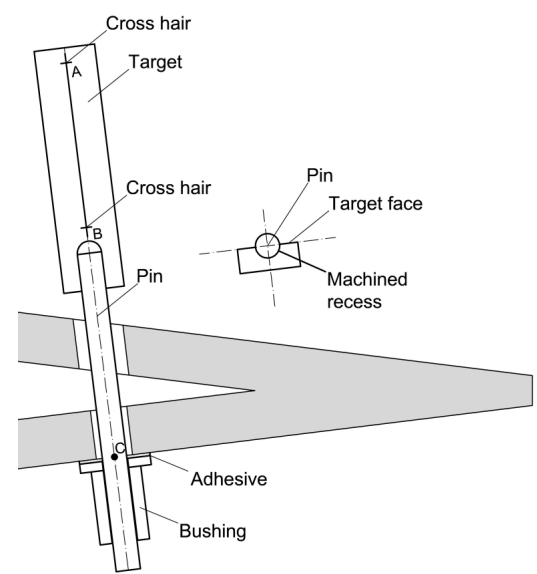

Figure 10: Pin and target adhesively connected to lower shell via height adjustable bushing

Neon light was used to enhance the contrast of the grid lines and the cross hairs of the target. In images with slightly blurred areas an edge detection filter was applied to identify the grid line border lines. The digital cameras of the SDMS were electronically modified such that they could be remotely focused and triggered simultaneously. With a set of images taken before and after deformation, the SDMS coordinates of the cross hairs (i.e. $\vec{A}$ and $\vec{B})$ can be measured. The LRD vector $\tilde{\vec{\omega}}$ in $3 \mathrm{D}$ space can then be consequently obtained with equation 5 .

\subsection{Estimating the SDMS precision}

In this section possible sources of measurement errors are discussed and the system accuracy of the SDMS is investigated. The pinhole camera model used by the software [8] represents a first-order approximation of maping a $3 \mathrm{D}$ scene onto a 2D image. In other words, a systematic error is introduced by higher order deviations of the pinhole camera model [4]. Since the tracking of pixels between subsequent images is still performed manually, measurements are also affected by subjective choices which depend on contrast, depth of field and light conditions. Figure 11 depicts an idealised magnification of an ill conditioned gridline intersection. It shows the difficulties associated with finding the precise intersection due to blurred and skew boundaries. In its current configuration depending on the location in the image the resolution of one grid line varies between three and 15 pixels. 


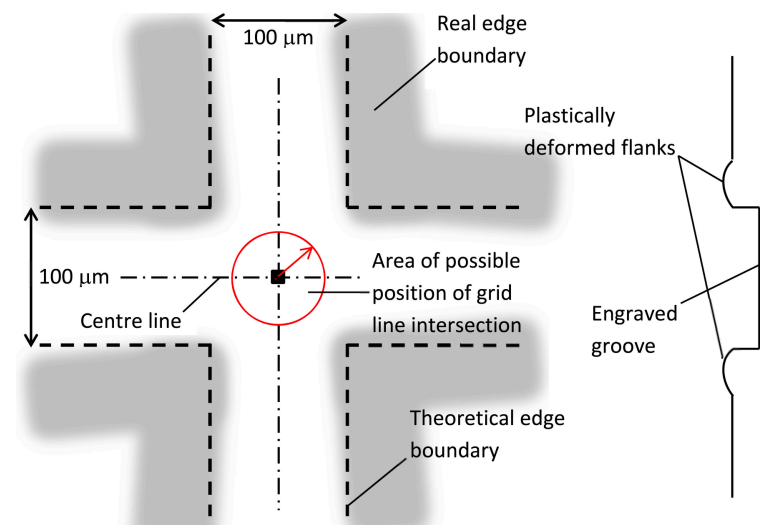

Figure 11: Grid line intersection issue

The SDMS was tested in two stages in order to obtain the accuracy of the system itself. First, the SDMS was used to predict the coordinates of the grid. This step served the purpose of finding the ideal number and appropriate location of calibration points on the grid. Secondly, this step aimed to establish the most accurate method for pixel selection at the intersections of the grid lines. Table 1 lists the true and the predicted real world coordinates of six different gridpoints. Statistical analysis showed that the differences given in columns eight through ten follow a normal distribution with a mean value of -0.17 microns and a standard deviation of $\sigma=13$ microns (corresponds to a thirteenth of the grid line width).

Table 1: Prediction of gridpoint coordinates

\begin{tabular}{lccccccccc}
\hline Gridpoint & $\begin{array}{c}X \\
{[\mathrm{~mm}]}\end{array}$ & $\begin{array}{c}Y \\
{[\mathrm{~mm}]}\end{array}$ & $\begin{array}{c}Z \\
{[\mathrm{~mm}]}\end{array}$ & $\begin{array}{c}X_{p} \\
{[\mathrm{~mm}]}\end{array}$ & $\begin{array}{c}Y_{p} \\
{[\mathrm{~mm}]}\end{array}$ & $\begin{array}{c}Z_{p} \\
{[\mathrm{~mm}]}\end{array}$ & $\begin{array}{c}d_{X} \\
{[\mathrm{~mm}]}\end{array}$ & $\begin{array}{c}d_{Y} \\
{[\mathrm{~mm}]}\end{array}$ & $\begin{array}{c}d_{Z} \\
{[\mathrm{~mm}]}\end{array}$ \\
\hline 1 (D4) & 20.000 & 35.000 & 10.080 & 20.008 & 34.982 & 10.074 & 0.008 & -0.018 & -0.006 \\
2 (E4) & 20.000 & 40.000 & 10.080 & 20.016 & 40.002 & 10.084 & 0.016 & 0.002 & 0.004 \\
3 (F4) & 20.000 & 45.000 & 10.080 & 20.012 & 44.993 & 10.085 & 0.012 & -0.007 & 0.005 \\
4 (G4) & 20.000 & 50.000 & 10.080 & 20.017 & 50.002 & 10.074 & 0.017 & 0.002 & -0.006 \\
5 (H4) & 20.000 & 55.000 & 10.080 & 20.018 & 54.994 & 10.042 & 0.018 & -0.006 & -0.038 \\
6 (G13) & 65.000 & 50.000 & 10.080 & 65.010 & 49.992 & 10.072 & 0.010 & -0.008 & -0.008 \\
\hline
\end{tabular}

where $X, Y, Z$ denote the real world gridpoint coordinates, $X_{p}, Y_{p}, Z_{p}$ represent the predicted gridpoint coordinates and $d_{X}, d_{Y}, d_{Z}$ are the differences between the true and the predicted gridpoint coordinates in the respective directions.

Performing this accuracy test for different numbers and locations of calibration points it was found that homographic calibration, using a single plane, gave highly inaccurate results. Instead good accuracy was obtained with 24 distributed calibration points located on three different planes of the grid. From gridpoint 5 it can be seen how blur, bad light conditions, reflections and manufacturing induced edge deformations cause a considerable 
inaccuracy in finding the true grid line intersection (see Fig. 11). The findings of the first step were used in a second step to measure the coordinates of the target displaced in pure translation. For this purpose the SDMS was mounted stationary and the target was moved by a 3-axis milling machine. The servo controlled milling machine has got a designated precision of \pm 10 microns. The target was subsequently moved by 50, 100, 500, 1000 and 5000 microns separately in all three directions. In Tab. 2 the first column represents the repeatedly imposed displacements where $\Delta x_{m}, \Delta y_{m}$, and $\Delta z_{m}$ represent the measured values and $d x, d y$ and $d z$ represent the differences between the imposed and the measured displacements.

Table 2: Measurement of imposed translations in $x, y$ and $z$ direction

\begin{tabular}{ccccccc}
\hline $\begin{array}{c}\text { Imposed } \\
{[\mathrm{mm}]}\end{array}$ & $\begin{array}{c}\Delta x_{m} \\
{[\mathrm{~mm}]}\end{array}$ & $\begin{array}{c}\Delta y_{m} \\
{[\mathrm{~mm}]}\end{array}$ & $\begin{array}{c}\Delta z_{m} \\
{[\mathrm{~mm}]}\end{array}$ & $\begin{array}{c}d x \\
{[\mathrm{~mm}]}\end{array}$ & $\begin{array}{c}d y \\
{[\mathrm{~mm}]}\end{array}$ & $\begin{array}{c}d z \\
{[\mathrm{~mm}]}\end{array}$ \\
\hline 0.050 & 0.051 & 0.051 & 0.079 & -0.001 & -0.001 & -0.029 \\
0.100 & 0.101 & 0.098 & 0.133 & -0.001 & 0.002 & -0.033 \\
0.500 & 0.496 & 0.513 & 0.529 & 0.004 & -0.013 & -0.029 \\
1.000 & 0.992 & 0.966 & 1.036 & 0.008 & 0.034 & -0.036 \\
5.000 & 4.969 & 4.999 & 5.036 & 0.031 & 0.001 & -0.036 \\
\hline
\end{tabular}

The values in Tab. 2 given in columns five through seven follow as well a normal distribution. The differences $d x$ and $d y$ have got a mean value of 6 microns and a standard deviation of $\sigma=15$ microns. However, the mean of $d z$ in column seven shows to have a mean value (i.e. consistent offset) of 33 microns which infers to a systematic error introduced by the milling machine control. The standard deviation of $d z$ is $\sigma=4$ microns.

\subsection{Blade testing methodology}

A standardised procedure for full scale testing of wind turbine blades can be found in [1]. However, the blade test presented in this paper was performed according to a non-standardised procedure. The blade had a length of $34 \mathrm{~m}$, a root diameter of $1.8 \mathrm{~m}$ and a net weight of $4500 \mathrm{~kg}$. The blade was truncated at $29 \mathrm{~m}$. The root of the blade was bolted to a rigid steel rig in such a way that the blade axis had an angle of $8^{\circ}$ with respect to the horizontal direction. The tilt of the blade axis increased the available tip displacement that could imposed on the blade.

The chord (i.e. shortest distance between the leading and the trailing edge in an airfoil) at the tip was tilted by an angle of $30^{\circ}$ with respect to the horizontal direction (see Fig. 12b). The blade was loaded at a horizontal distance of $28.4 \mathrm{~m}$ by a hydraulic winch (see Fig. 12a). The winch was fastened to steel frames which were connected to the floor. The load was introduced through a displacement controlled mechanism that pulled the blade vertically towards the floor via pulleys. The load magnitude was measured by an interconnected load cell. The wire rope of the winch was fastened to a steel loading plate that was adhesively connected to the suction side 


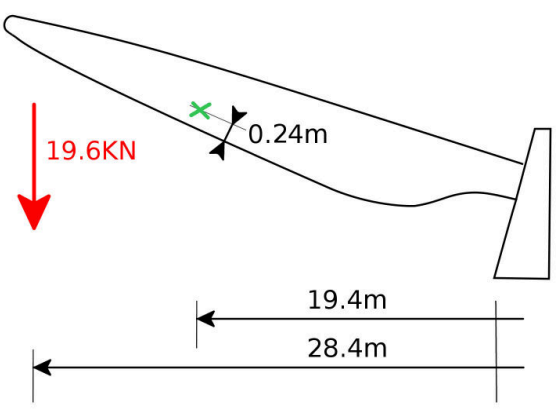

(a)

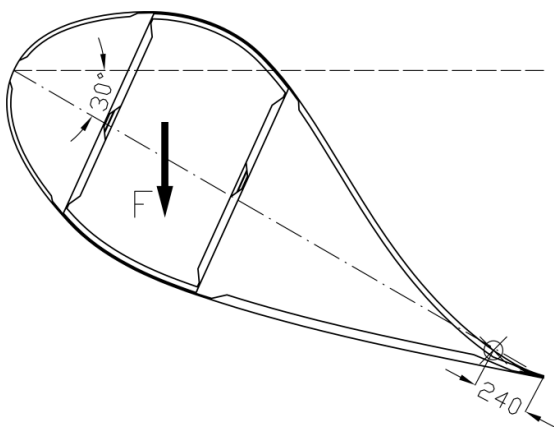

(b)

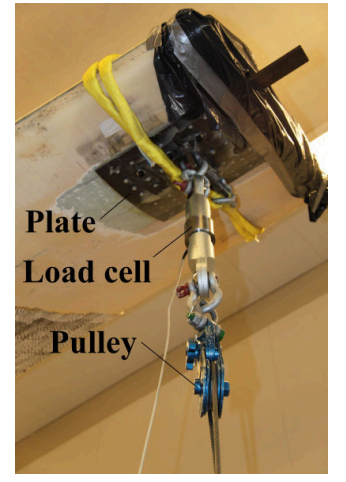

(c)

Figure 12: Schematic experimental setup (a) $19.4 \mathrm{~m}$ airfoil (b) tip load application point (c)

shell (see Fig. 12c). The load introduction point was positioned in such a way that the line of action intersected the shear centre of the cross-section. Additional blind bolts were used for the plates to increase the connection strength. The test performed is referred to as a combined test because it combines flapwise and edgewise bending. The load was applied quasi-statically up to a maximum load level of $19.60 \mathrm{kN}$.

\subsection{Instrumentation}

The SDMS was located on the pressure side shell at a distance of $19.4 \mathrm{~m}$ from the blade root (see Fig. 12a). The frame of the SDMS was adhesively connected to the pressure side shell of the blade via a small aluminium foot with a square base plate. Small screws were used to reinforce the adhesive connection (see Fig. 13). Referring to Fig. 9 the X-Z-plane of the grid was tangentially aligned with the shell surface (Y-axis $\left.\widehat{=} \overrightarrow{n_{1}}\right)$ with the Z-axis being parallel to the trailing edge. In this setup LRDs in the X-direction correspond to Mode-II, displacements in the Y-direction to Mode-I and those in the Z-direction to Mode-III. The pin/target was adhesively connected to the suction side shell by a steel bushing which allowed height adjustments to be made. The pin holes in the shells were located $0.24 \mathrm{~m}$ away from the trailing edge and about $0.12 \mathrm{~m}$ away from the flow front of the adhesive joint.

An additional independent measurement was performed adjacent to the SDMS target. A Novotechnik TRS 50 linear transducer (LVDT) with an accuracy of \pm 100 microns was mounted perpendicularly to the pressure side shell. A small hole in the pressure side shell enabled the transducer head to rest on the inner surface of the lower shell. In this way the Mode-I opening displacement could be measured (see Fig. 14). 


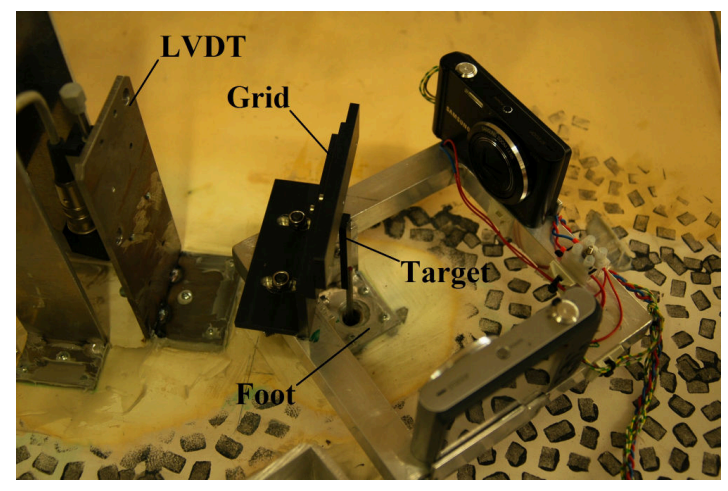

(a)

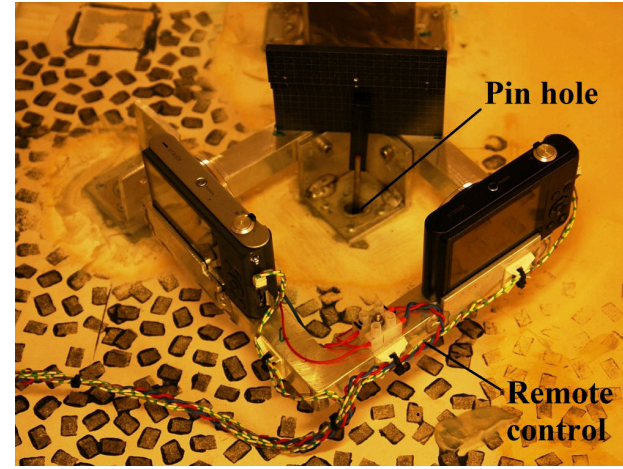

(b)

Figure 13: SDMS and LVDT mounted on the pressure side shell

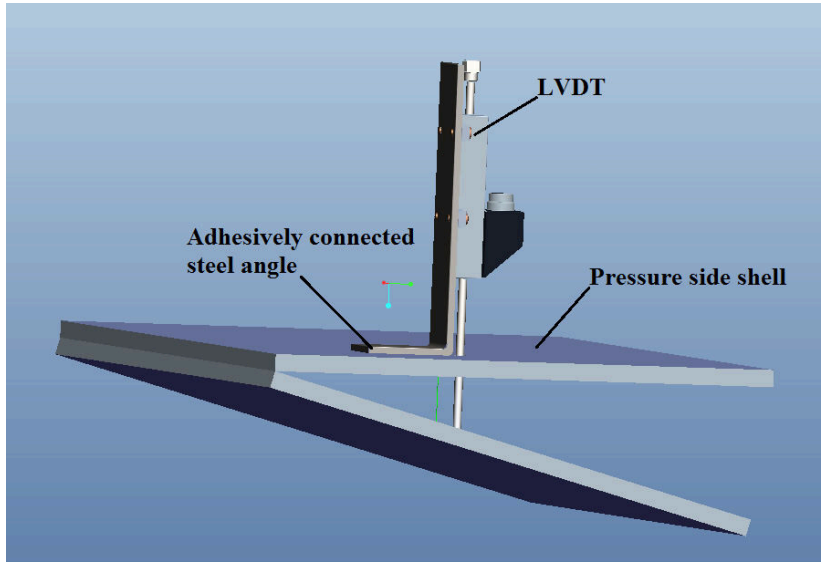

Figure 14: Additional instrumentation adjacent to the SDMS

\section{Results}

After the maximum load level of $19.6 \mathrm{kN}$ was reached the load level was maintained for some minutes before measurements were taken. This procedure allowed the decay of oscillations induced by load redistribution effects. The blade test was subsequently repeated three times in order to ensure that the measured data was consistent. Figure 15 shows two images taken from the same camera showing the target position before and after local deformation. In the image the gridlines appear to be straight with negligible distortion. Vectors $\vec{a}=\overrightarrow{A^{\prime}}-\vec{A}$ and $\vec{b}=\vec{B}^{\prime}-\vec{B}$ represent the measured displacements (in millimetres) of the target points in SDMS coordinates. Vector $\tilde{\vec{\omega}}$ represents the LRD in intermediate plane coordinates according to equation 5 . Vector $\vec{\omega}$ represents the LRD in SDMS coordinates. 


$$
\begin{aligned}
\vec{a} & =\left\{\begin{array}{lll}
-0.531 & -0.734 & -0.036
\end{array}\right\}^{T} \\
\vec{b} & =\left\{\begin{array}{lll}
-0.344 & -0.744 & -0.025
\end{array}\right\}^{T} \\
\tilde{\vec{\omega}} & =\left\{\begin{array}{lll}
-0.269 & -0.640 & -0.019
\end{array}\right\}^{T} \\
\vec{\omega} & =\left\{\begin{array}{lll}
0.245 & -0.774 & 0.009
\end{array}\right\}^{T}
\end{aligned}
$$

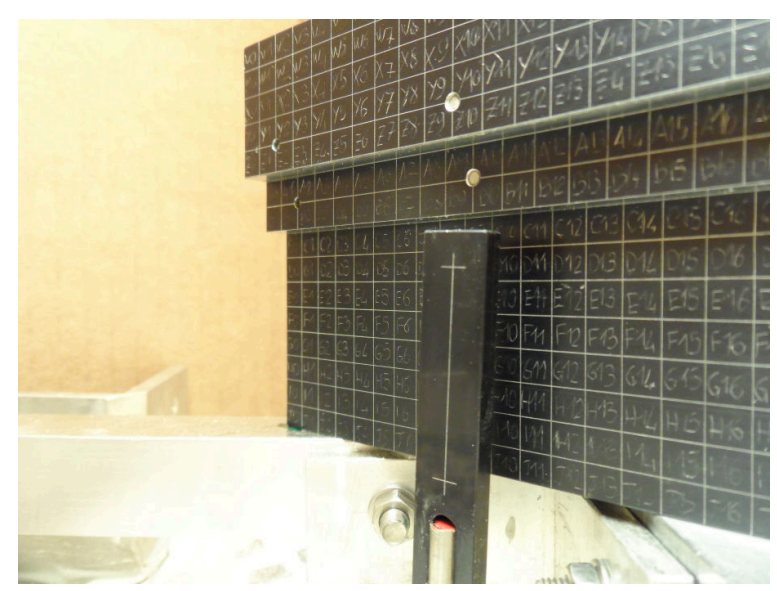

(a)

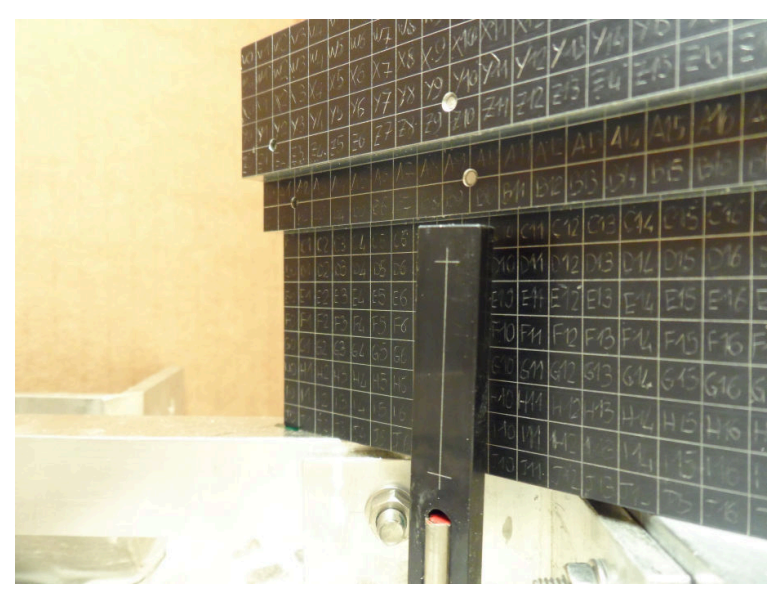

(b)

Figure 15: Grid with target undeformed configuration (a) and deformed configuration (b)

The vector $\tilde{\vec{\omega}}$ suggests that the LRDs were governed by Mode-I closing of $-0.640 \mathrm{~mm}$. A comparatively small component of $-0.269 \mathrm{~mm}$ could be measured in the Mode-II direction where the Mode-III component yields a value of $-0.019 \mathrm{~mm}$. Figure 16 shows the LVDT displacements plotted against the applied tip load magnitude for the loading and unloading situation. Although some noise in the measured signal is present, the loading and unloading paths are reasonably well aligned which confirms the repeatability of the measurements. The peaks seen in Fig. 16 which consistently appear in the loading and unloading path, are caused by load redistribution effects which have been observed before in wind turbine blade tests. Moreover it can be seen that the LVDT measured a Mode-I closing of $0.8 \mathrm{~mm} \pm 0.1 \mathrm{~mm}$ at the maximum tip load level of $19.6 \mathrm{kN}$. In order to be comparable with the LVDT measurement the Mode-I component of the LRD in SDMS coordinates needs to be considered where the latter yields a closing of $-0.774 \mathrm{~mm}$. 


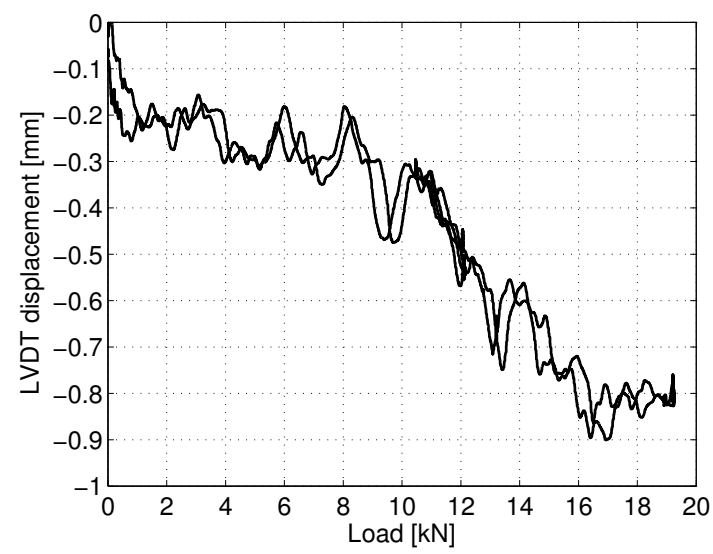

Figure 16: LVDT displacements versus tip load magnitude

\section{Discussion}

The Mode-I LRD results presented in Section 3 deviated $3 \%$ and show good agreement. The position of the measurement equipment was chosen far enough away from the trailing edge as to ensure that the displacement level was within the accuracy of the LVDT. Because of its higher accuracy the SDMS can be mounted closer to the flow front of the adhesive joint which would increase the significance of the measurements with regard to fracture mechanics.

The measurement of LRDs between two opposite points located on the trailing edge shells represents a challenge. The difficulty is caused by determining the RBMs which represent a virtual quantity. All known approaches that aim at measuring LRDs depend on assumptions to be made about the rigid body motions. That is, either implicitly by assuming that the measurement equipment follows the RBM or explicitly by subtracting the RBM from the total deformations. It is likely that the mounting device of the SDMS (e.g. hole diameter, plate size and thickness, adhesive type and thickness, etc.) can have some influence on the local deformation behaviour. Furthermore, deviations concerning the assumption that the SDMS is rigidly connected and follows the tangential movement in one point can be expected. Nevertheless, measurements with the SDMS can still be used for comparison with other measurements if the chosen method is consistently applied. Furthermore, measurements can be used for comparison with FE-results if the local stiffness of the mounting method is taken into consideration. From that it can be inferred that the proposed method is a starting point to experimentally investigate fracture behaviour in large structures. Accuracy tests showed that a reference grid produced by engraving lines into a surface potentially introduces inaccuracies. The adoption of a different grid pattern (e.g. checkerboard) can mitigate this issue and increase the camera calibration accuracy.

A thorough interpretation of the measurement results is beyond the scope of this paper. Moreover, a greater 
number of measurements would be required to decrease uncertainties. The measured Mode-III deformations as discussed in [5] were too small in this case to serve for comparison purposes. On the other hand, the applied shear force was comparatively low, leading to low out-of-plane shear displacements. However, the Mode-I closing and Mode-II shear displacements of the shells can be caused by geometric nonlinearity effects or by layup asymmetry. Furthermore, abrupt changes of the composite layup thickness cause local discontinuities in the shell which lead to deviations of the membrane stress state. These deviations manifest themselves in localised bending deformations that induce mixed mode conditions that can overrule the significance of the Mode-III response.

Future research will focus on the development of a digital image correlation algorithm with an automatic pixel tracking routine. Such an automated procedure would enable a higher number of measurements to be taken efficiently at different locations in order to gain more insight into the trailing edge deformation behaviour. Future work also involves the development of a real-time measurement technique which could be used to monitor the behaviour of structures under operating loading conditions.

\section{Conclusion}

Following the discussion the following conclusions can be drawn:

(i) To the author's best knowledge there is currently no accurate and unbiased LRD measurement method available that can be applied in a similar manner as the one proposed.

(ii) The proposed method represents a cost efficient and straight forward alternative to measure the three components of LRD vector in trailing edge joints of wind turbine blades.

(iii) The method can generally be applied to other kinds of adhesive joints in large structures that undergo large global deformations.

(iv) The SDMS in its current configuration is able to provide an accuracy of \pm 15 microns.

(iv) The measured LRDs as a result of the applied loading condition were governed by Mode-I closing and Mode-II (in-plane) shear.

(v) Digital image correlation with a sufficient accuracy for application in engineering practice can be achieved with off-the-shelf digital cameras. 


\section{Acknowledgements}

The authors would like to acknowledge the advice and support that Peter Berring provided for validating the results. Many thanks to Helmuth L. Toftegaard and Kim Branner for their input and advise. The authors are grateful for the courtesy of Claus B. M. Pedersen (DTU-TEM programme) for performing all the electronic modifications. The authors are much obliged to the efforts of Christian Lyngbæk (DTU workshop) for his commitment and patience in manufacturing the mechanical parts of the system and for the tedious preparations of the full scale blade test. The authors would like to thank Basel Hayatleh (DTU-VIM) for providing the 3D illustrations. The work is supported by the Danish Energy Agency through the 2010 Energy Technology Development and Demonstration Program (EUDP). The supported EUDP-project titled is "Experimental Blade Research - Phase 2" and has journal no. 64011-0006. The support is gratefully acknowledged.

\section{References}

[1] IEC 61400-23. Full-scale structural testing of rotor blades, 2012.

[2] T. L. Anderson. Fracture Mechanics - Fundamentals and Applications. Taylor and Francis, 2005.

[3] S. Ataya and M. M. Z. Ahmed. Damages of wind turbine blade trailing edge: Forms, location, and root causes. Engineering Failure Analysis, in press, 2013.

[4] K. B. Atkinson. Close Range Photogrammetry and Machine Vision. Whittles Publishing, 1996.

[5] M. E. Eder, Bitsche R. D., M. Nielsen, and Branner K. A practical approach to fracture analysis at the trailing edge of wind turbine rotor blades. Wind Energy, 2013.

[6] V. Fedorov. Bend-Twist Coupling Effects in Wind Turbine Blades. Dissertation, Technical University of Denmark, 2012.

[7] GOM. ARAMIS User Manual - Software, ARAMIS v6.1. GOM Gmbh, Braunschweig Germany, 2008.

[8] P. Sheer. A Software Assistant for Manual Stereo Photometrology. Msc thesis, University of the Witwatersrand, 1997.

[9] B. F. Sørensen and T. K. Jacobsen. Characterizing delamination of fibre composites by mixed mode cohesive laws. Composites Science and Technology, 69:445-456, 2009.

[10] S. P. Timoshenko. On the correction factor for shear of the differential equation for transverse vibrations of bars of uniform cross section. Philosophical Magazine, page 744, 1921. 


\section{Appendix}

\section{The significance of $\Pi_{0}$ and $R$}

Figure 17 a shows a $2 \mathrm{D}$ example of two initially parallel lines which means that $P 1$ and $R$ coincide. In Case (a) the two lines simultaneously rotate by the same angle in the same direction. Consequently the bisector (indicated by a dash-dotted line) remains parallel to the solid lines and the LRD between points $P 2$ and $R$ can directly be obtained in SDMS coordinates according to $\vec{\omega}=\tilde{\vec{\omega}}=\vec{r}^{\prime}-\vec{r}$.
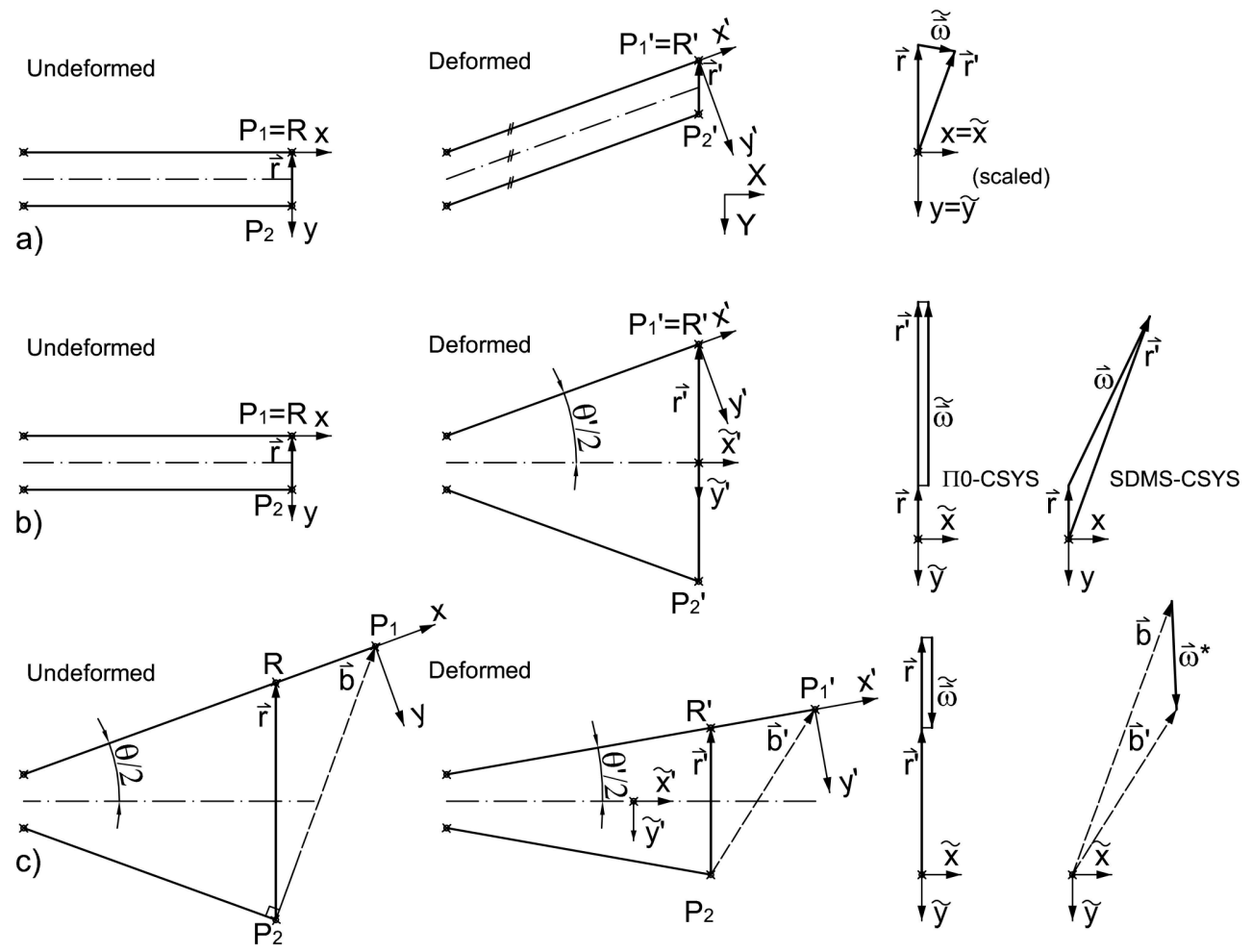

Figure 17: Three different 2D LRD cases showing the importance of bisector transformation and the role of the true point

The rotation of the $\Pi_{0}$ cordinate system into its primed position represents the RBM. It can also be seen from Fig. 17a that the applied motion leads to Mode-I closing and Mode-II shearing which cannot be directly obtained from a global coordinate system (indicated by capital letters). The latter wrongly results in zero LRD since the moduli of $\vec{r}$ and $\vec{r}^{\prime}$ remain constant for this deformation mode.

Figure $17 \mathrm{~b}$ shows the same initial configuration as mentioned above. In Case (b) the lines rotate by the same angle in different directions which corresponds to a pure Mode-I opening. It can be seen from Fig. 17b that the LRD in SDMS coordinates spuriously predicts a Mode-II component. In order to correctly obtain the LRD the 
coordinate system needs to undergo a transformation into the $\Pi_{0}$ coordinate system.

Figure 17c shows two initially non-parallel lines where $P 1$ and $R$ do not coincide. In Case (c) the lines rotate by the same angle in different directions which corresponds to a pure Mode-I closing. It can be seen from Fig. 17c that $\vec{\omega}^{*}$ spuriously predicts a Mode-II component. Conversely, the adoption of the true point leads to the correct LRD. It needs to be mentioned that the deviations for small values of $\theta$ as they appear in trailing edges remote from the blade root close to the bond line are also small. 